\title{
Application of management guidelines to the assessment of new medicines: driving us forward or holding us back?
}

\author{
P.M.A. Calverley*, S.I. Rennard ${ }^{\#}$
}

\begin{abstract}
Clinical management guidelines have made an invaluable contribution in many areas of medicine. By providing recommendations based on an expert assessment of the available evidence, guidelines can identify "best practice" approaches, help drive consistent quality of care, reduce morbidity and mortality, and improve cost-effectiveness. The considerable time, effort and organisation needed to prepare authoritative sets of guidelines provides an imperative to gain the maximum utility from their development. However, it is also important that guidelines are used appropriately. The use of guidelines to address issues other than those for which they were designed can potentially have a detrimental effect on patient care.
\end{abstract}

In chronic obstructive pulmonary disease (COPD), for instance, guidelines from bodies such as the Global Initiative for Chronic Lung Disease (GOLD) [1], the European Respiratory Society (ERS) [2] and the American Thoracic Society (ATS) [3] have helped to establish standards of care, and help drive improved management of COPD. However, the guidelines have also been used increasingly by regulatory authorities to define the requirements for new agents to gain approval, a role that they were never designed to fill.

The GOLD guidelines provide a working definition of COPD as a disease state characterised by airflow limitation, which is not fully reversible, and is usually both progressive and associated with an abnormal inflammatory response to noxious particles [1]. They also, however, recognise both the heterogeneity of COPD and the difficulties of providing a comprehensive definition for a disease state with such a variable natural history. Airflow limitation is also used to categorise COPD according to disease severity, although the guidelines again emphasise this is intended as a practical operational and educational tool [1]. Despite this, regulatory authorities have used the definitions of COPD within the guidelines to define the requirements for approval of new agents and so shape the design of clinical trials [4].

As an example, European regulatory agencies require that patients enrolled in clinical trials for COPD medications show a limited response to bronchodilators, in an attempt to exclude patients with a primary diagnosis of asthma. This is particularly problematic as COPD and asthma may coexist, and recent data suggest that asthma can progress to COPD in some cases. This artificial definition was developed for the purposes of clinical trials studying the natural history of COPD and the effect of treatment intervention. Its utility in rigorously differentiating a "COPD" from an "asthma" phenotype has not been tested prospectively and would probably

*Clinical Science Centre, The University Hospital Aintree, Liverpool, UK and ${ }^{\#}$ Dept of Internal Medicine, University of Nebraska Medical Center, Omaha, NE, USA.

Correspondence: P.M.A. Calverley, Dept of Medicine, The University Hospital Aintree, Clinical Science Centre, Longmoor Lane, Liverpool L9 7AL, UK. Fax: 44 1515292931. E-mail: pmacal@liverpool.ac.uk fail a rigorous test. The limitations of this approach have been highlighted in a recent analysis of data from the Inhaled Steroids in Obstructive Lung Disease in Europe (ISOLDE) study [5]. The analysis indicates that, even among the patients with COPD defined as "poorly reversible" who were recruited to the ISOLDE study, there was a continuum of responsiveness to bronchodilators. Furthermore, on repeat bronchodilator testing at separate visits, a large proportion of patients changed classification from bronchodilator "responders" to "nonresponders", or vice versa (52.1\% using ATS criteria, 38.2\% using ERS criteria) [5]. As the classification of patients as "responders" or "nonresponders" is arbitrary and not predictive of future bronchodilator response [5], there is little logic for excluding, from clinical trials, patients presenting with a clinical picture typical of COPD but who show, or fail to show, an arbitrarily defined bronchodilator response at a particular point in time [6]. What the ISOLDE analysis does support is the judgement of the doctor who labelled the patient as having COPD rather than asthma and confirmed the diagnosis with spirometry without reversibility testing.

Regulatory bodies have taken a similar approach with asthma. The Committee for Proprietary Medicinal Products (CPMP) of the European Agency for the Evaluation of Medicinal Products has framed its guidance on trials of asthma medications around the Global Initiative for Asthma (GINA) guidelines, including the classification of asthma into four grades of severity. In particular, the CPMP indicates that "it is expected that separate trials for a new product be carried out for each grade of asthma severity. The claimed indication should only include those grades of asthma severity in which the new drug has been tested and found to have a favourable risk/benefit ratio" [7]. Despite not being designed or validated for the purpose, the GINA gradings are being used to shape the regulatory requirements for new asthma medications. Indeed, the CPMP guidance observes that the relevance of the GINA gradings is primarily in the initial assessment of patients and that other classifications may be more relevant for patients well controlled on their medication [7].

The inappropriate use of guidelines by regulatory bodies generates a circularity that threatens to stifle progress in the management of COPD. Guideline committees base their recommendations, in large part, on the evidence from large, randomised trials, most of which are sponsored by pharmaceutical companies to support registration of their products. The outcomes used in these studies will, therefore, reflect the requirements of regulatory authorities. If the regulatory authorities, in turn, base their requirements on the guidelines, current practices become entrenched. Potentially valuable new approaches to treatment may be missed, essentially because they work in different ways to established therapies.

Regulatory bodies have to make difficult decisions to ensure that licensed medicines are both safe and efficacious. 
This is much easier when there is professional agreement about what defines the disease under study, such as an elevated low-density cholesterol level or a visible duodenal ulcer at endoscopy. The syndromic approach adopted when defining COPD and asthma makes for many more "grey areas". The individual treatment guidelines do not specify upper or lower limits for bronchodilator responsiveness, and certainly do not define either asthma or COPD purely by the short-term response to a single inhalation of a bronchodilator drug of unspecified nature and dose. Inclusion of this definition in the requirements for clinical studies by the European regulators not only extends this process well beyond its original intent but calls into question studies admissible for licensing by the US Food and Drug Administration, which does not require a specified degree of reversibility to be present. As a result, some meta-analyses addressing the role of therapy can omit most of the published data and reach rather surprising conclusions [8]. It is important that the medical profession and the regulatory agencies develop a continuing dialogue to address these issues, especially given recent changes in the understanding of these diseases.

Current treatment options need to be supplemented with new agents and strategies if the growing burden of morbidity and mortality, due to chronic obstructive pulmonary disease, are to be countered. The inappropriate use of management guidelines in the regulatory environment has the potential to suppress the introduction of new and potentially valuable treatments. Paradoxically, there is a danger that treatments that could advance the management of chronic obstructive pulmonary disease are rejected precisely because they act in novel ways and have different effects from established treatments. The assessment of new drugs needs to have an eye to the future, as well as applying the lessons of the past.

\section{References}

1. Global Initiative for Chronic Obstructive Lung Disease. Global strategy for the diagnosis, management and prevention of chronic obstructive pulmonary disease. NHLBI/ WHO Workshop Report 2001, updated 2003. http://www. goldcopd.com. Date last updated: July, 2003. Date last accessed: March 2004.

2. Siafakas NM, Vermeire P, Pride NB, et al. Optimal assessment and management of chronic obstructive pulmonary disease (COPD). The European Respiratory Society Task Force. Eur Respir J 1995; 8: 1398-1420.

3. American Thoracic Society. Standards for the diagnosis and care of patients with chronic obstructive pulmonary disease. Am J Respir Crit Care Med 1995; 152: S77-S121.

4. Committee for Proprietary Medicinal Products (CPMP) Points to consider on clinical investigation of medicinal products in the chronic treatment of patients with chronic obstructive pulmonary disease (COPD). European Agency for the Evaluation of Medicinal Products, 1999 May. Report No: $562 / 98$.

5. Calverley PM, Burge PS, Spencer S, Anderson JA, Jones PW. Bronchodilator reversibility testing in chronic obstructive pulmonary disease. Thorax 2003; 58: 659-664.

6. Gross NJ. Responses to steroids and bronchodilators in COPD in the ISOLDE trial: the fat lady sings on. Thorax 2003; 58: 647-648.

7. Committee for Proprietary Medicinal Products (CPMP). Note for guidance on the clinical investigation of medicinal products in the treatment of asthma. European Agency for the Evaluation of Medicinal Products, 2002 Nov. Report No: $2922 / 01$

8. Appleton S, Smith B, Veale A, Bara A. Long-acting $\beta_{2}$-agonists for chronic obstructive pulmonary disease. Cochrane Database Syst Rev 2000; 2: CD001104. 